\title{
ERROR ESTIMATES FOR A NONLINEAR DEGENERATE PARABOLIC PROBLEM
}

\author{
P. LESAINT AND J. POUSIN
}

\begin{abstract}
In this paper we are dealing with a partial differential equation of parabolic type, which degenerates on one side of the domain. This equation may be viewed either as a model of particle diffusion in plasma physics, or as a simplified model of a viscous boundary layer in two dimensions. Known results for the existence and uniqueness of the weak solution are first recalled. A finite difference implicit scheme is then defined, and error bounds are derived, taking into account the low degree of smoothness of the exact solution. An iterative algorithm for the computation of the numerical solution at each time step is shown to be convergent.
\end{abstract}

\section{INTRODUCTION}

The problem that we intend to study is the modeling of diffusion of particles (see Drake et al. [9, equation $\left.A_{1}\right]$ ) in plasma physics, where the unknown function $u$ denotes the density of the particles.

This problem may also be viewed as a simplification of the equations for a viscous boundary layer in two dimensions, occurring in fluid mechanics in the case of an incompressible fluid flow around a solid body. If we neglect the advection terms in the direction orthogonal to the body, the unknown function $u$ represents the velocity of the fluid along the $t$ direction, parallel to the body, and the other variable $x$ represents the distance to the body (see also Oleinik [18]). Let $Q_{T}=(0,1) \times(0, T)$; we define the problem $(\mathrm{P})$ as follows:

$$
\begin{array}{ll}
\text { to find } u(x, t) \text { satisfying: } & \\
u u_{t}-u_{x x}=0 & \text { in } Q_{T}, \\
u(x, 0)=u_{0}(x) & \text { in }(0,1), \\
u(0, t)=0, u(1, t)=u_{1}(t) & \text { in }(0, T) .
\end{array}
$$

Our main results concerning problem (P) are the following: a finite difference implicit scheme is defined, existence and uniqueness of the numerical solution is proved, and error bounds are derived. An iterative algorithm allowing us to compute the numerical solution at each time step is defined, and the convergence is proved.

Received by the editor December 1, 1989 and, in revised form, September 3, 1990 and August 13, 1991.

1991 Mathematics Subject Classification. Primary 35K65, 65M15, 65M06, 65M12.

Key words and phrases. Numerical analysis, nonlinear, parabolic equation, finite differences, error estimates.

This work is partially supported by the European Hermès project and by the EPFL Lausanne. 
An outline of the paper is as follows. The later part of this section is devoted to the assumptions concerning problem $(\mathrm{P})$. A weak formulation of this problem is given and known results for the existence and uniqueness of the weak solution are recalled. In $\S 2$, we define a finite difference scheme, implicit with respect to the time variable $t$. We show the existence, uniqueness, and stability of the numerical solution. In $\S 3$, we adapt a technique first developed for the Stefan problem by Nochetto [16], and we derive an estimate of the error between the exact solution $u$ and the approximate solution $U$, namely

$$
\|U-u\|_{L^{2}\left(Q_{T}\right)} \leq C\left(\frac{\tau}{h}+h^{1 / 2}\right),
$$

where $\tau$ and $h$ denote the parameters of the discretization respectively in time and in space. The term $\frac{1}{h}$ arising in the estimate is the price to pay for the degeneration of the problem at $x=0$. In $\S 4$, we define an iterative algorithm which allows us to solve the nonlinear system of equations satisfied by the approximate solution at each time step. We show that this algorithm converges when the number of iterations goes to infinity. In the last section, we give some possible generalizations of the previous results in the cases where the term $u u_{t}$ is replaced by $u^{p-1} u_{t}, p \geq 2$, and (or) for a spatial dimension equal to two or three.

We now recall results of existence and uniqueness for the weak solution of problem (P); a proof of these results can be found, for example, in Sabinina [20] or in [19]. Other results of existence and uniqueness of an even weaker solution have been derived; for these we refer to Arison and Benilan [1], Arison and Peletier [2, pp. 381-411], Benilan [3], and Herrero and Pierre [12].

We define the following spaces:

$$
\begin{gathered}
V_{2}^{1,0}\left(Q_{T}\right)=C^{0}\left(0, T ; L^{2}(0,1)\right) \cap L^{2}\left(0, T ; H^{1}(0,1)\right) \\
W_{2}^{1,1}\left(Q_{T}\right)=\left\{\varphi \in H^{1}\left(Q_{T}\right) ; \varphi(0, t)=\varphi(1, t)=0,0<t<T ;\right. \\
\varphi(x, T)=0,0<x<1\}
\end{gathered}
$$

and we denote by $\|\varphi\|_{p ; Q_{T}}$ the usual norm in $L^{p}\left(Q_{T}\right), 1 \leq p \leq \infty$.

We assume that the following hypotheses are satisfied:

HP1. $u_{0}(x) \in C^{0}([0,1]), u_{1} \in C^{0}([0, T])$ and have bounded derivative;

HP2. $u_{1}(0)=u_{0}(1), u_{0}(0)=0$;

HP3. $u_{0}$ and $u_{1}$ are positive functions and $\exists \beta>0$ such that $u_{0}(x) \geq \beta x$ for $0 \leq x \leq 1$ and $u_{1}(t) \geq \beta$ for $0 \leq t \leq T$.

A weak solution of problem $(P)$ is defined by:

Definition 1.1. The function $u \in V_{2}^{1,0}\left(Q_{T}\right)$ is a weak solution of problem (P) if

$$
\begin{aligned}
\int_{0}^{1} & -u_{0}^{2}(x) \varphi(x, 0) d x \\
& +\int_{Q_{T}} 2 u_{x}(x, t) \varphi_{x}(x, t)-u^{2}(x, t) \varphi_{t}(x, t) d x d t=0
\end{aligned}
$$

$\forall \varphi \in W_{2}^{1,1}\left(Q_{T}\right) \cap C^{0}\left(0, T ; L^{\infty}(0,1)\right) ; u(0, t)=0, u(1, t)=u_{1}(t), 0<t<T$.

We have the following result: 
Theorem 1.1. Problem (P) has a unique weak solution $u \in V_{2}^{1,0}\left(Q_{T}\right)$. Moreover,

(i) $u \in C^{0}\left(\overline{Q_{T}}\right) \cap L^{\infty}\left(0, T ; W^{1, \infty}(0,1)\right)$;

(ii) $\forall z>0, \int_{z}^{1} \int_{0}^{T}\left(u_{t}\right)^{2} d x d t \leq C / z$, where the constant $C$ is independent of $z$;

(iii) $\exists \beta>0$ such that $u(x, t) \geq \beta x$ in $\overline{Q_{T}}$.

Remark 1.1. For the sake of simplicity, we assume hereafter that $u_{1}(t)=b$ is independent of $t$. In the case where $u_{1}(t)$ is not a constant, there would arise boundary terms.

Proof of Theorem 1.1. The proof of such a theorem is now classical; see, for example, $[1,2]$ or $[20]$. We only sketch the idea of the proof, especially to obtain the estimate (ii).

We solve, for $\varepsilon>0$, the problem:

$$
\begin{array}{ll}
u^{\varepsilon} u_{t}^{\varepsilon}-u_{x x}^{\varepsilon}=0 & \text { in } Q_{T}, \\
u^{\varepsilon}(x, 0)=u_{0}(x)+\varepsilon & \text { in }(0,1), \\
u^{\varepsilon}(0, t)=\varepsilon, u^{\varepsilon}(1, t)=u_{1}(t)+\varepsilon & \text { in }(0, T) .
\end{array}
$$

It is known that such a problem, for any $\varepsilon>0$, admits a classical positive solution $u^{\varepsilon}$ satisfying $u^{\varepsilon} \geq \beta x$ (cf. $[13,11,15]$ ). To get the estimate (iii) for $u^{\varepsilon}$, just use the maximum principle on the linear equation:

$$
\begin{array}{ll}
u^{\varepsilon} e_{t}-e_{x x}=0, & \text { with boundary and initial conditions, } \\
& \text { where } e=u^{\varepsilon}-\beta x .
\end{array}
$$

To get the estimate (ii) for $u^{\varepsilon}$, we first multiply equation (1.2) by $u_{t}^{\varepsilon}$ and integrate in space and time. We obtain

$$
\begin{aligned}
\int_{0}^{T} & \int_{0}^{z} u^{\varepsilon}\left(u_{t}^{\varepsilon}\right)^{2} d x d t+\int_{0}^{T} \int_{z}^{1} u^{\varepsilon}\left(u_{t}^{\varepsilon}\right)^{2} d x d t \\
& +\frac{1}{2} \int_{0}^{1}\left(\left(u_{x}^{\varepsilon}(x, T)\right)^{2}-\left(u_{x}^{\varepsilon}(x, 0)\right)^{2}\right) d x=0 .
\end{aligned}
$$

Since $u^{\varepsilon}$ is positive and $u^{\varepsilon} \geq \beta x$, we easily get

$$
z \int_{0}^{T} \int_{z}^{1}\left(u_{t}^{\varepsilon}\right)^{2} d x d t \leq C .
$$

Taking the limit when $\varepsilon \rightarrow 0$, we get Theorem 1.1.

Remark 1.2. We have (cf. [4]):

for every $t_{0}, t_{1}, 0<t_{0}<t_{1}<\infty$,

$$
u_{t} \in L^{p}\left((0,1) \times\left(t_{0}, t_{1}\right)\right) \text { for any } 1 \leq p<\frac{3}{2} .
$$

2. THE DISCRETE PROBLEM, EXISTENCE AND UNIQUENESS OF A SOLUTION, AND A PRIORI ESTIMATES

For given integers $N>0$ and $m>0$, we let

$$
\begin{aligned}
& \tau=\frac{T}{N}, \quad t_{n}=n \tau, \quad 0 \leq n \leq N ; \\
& h=\frac{1}{m+1}, \quad x_{i}=i h, \quad 0 \leq i \leq m+1 .
\end{aligned}
$$


For $c$ a given constant, we define the spaces $V_{h}, V_{h}(c)$, and $W_{h}^{\tau}(c)$ by:

$$
\begin{aligned}
& V_{h}=\left\{\varphi(s) \in C^{0}[0,1] ;\left.\varphi\right|_{\left[x_{i}, x_{i+1}\right]} \text { is a polynomial of degree } 1\right\}, \\
& V_{h}(c)=\left\{\varphi \in V_{h} ; \varphi(0)=0, \varphi(1)=c\right\}, \\
& W_{h}^{\tau}(c)=\left\{\varphi(\cdot, \cdot) \in L^{2}\left(0, T ; C^{0}([0,1])\right) ;\left.\varphi\right|_{\left(t_{n-1}, t_{n}\right]}=\varphi^{n} \in V_{h}(c),\right. \\
& \left.1 \leq n \leq N, \text { where } \varphi^{n} \text { is constant with respect to } t\right\} .
\end{aligned}
$$

To the scalar product $(\cdot, \cdot)$ and norm $\|\cdot\|$ in $L^{2}(0,1)$ we associate respectively the discrete semiscalar product $(\cdot, \cdot)_{h}$ and seminorm $\|\cdot\|_{h}$ defined by

$$
\begin{gathered}
(u, v)_{h}=h \sum_{i=1}^{m} u\left(x_{i}\right) v\left(x_{i}\right)+\frac{h}{2}\left(u\left(x_{0}\right) v\left(x_{0}\right)+u\left(x_{m+1}\right) v\left(x_{m+1}\right)\right), \\
\|v\|_{h}^{2}=(v, v)_{h} \quad \forall u, v \in C^{0}([0,1]) .
\end{gathered}
$$

The function $\gamma$ from $\mathbb{R}$ to $\mathbb{R}^{+}$is defined by $\gamma(s)=\frac{1}{2} s^{2}$.

We define the approximate problem $\left(\mathbf{P}_{h}^{\tau}\right)$ as follows:

$$
\begin{aligned}
& \text { to find }\left\{U^{n}\right\}_{n=0}^{N} \in\left(V_{h}(b)\right)^{N+1}, U^{n} \geq 0, \text { such that } \\
& \left(\gamma\left(U^{n+1}\right)-\gamma\left(U^{n}\right), \varphi_{h}\right)_{h}+\tau a\left(U^{n+1}, \varphi_{h}\right)=0 \\
& \forall \varphi_{h} \in V_{h}(0), 0 \leq n \leq N-1, \\
& U^{0}=r_{h} u_{0}, \\
& \text { where } a(\varphi, \psi)=\int_{0}^{1} \varphi_{x} \psi_{x} d x,
\end{aligned}
$$

and where the interpolation operator $r_{h}$ in $V_{h}$ is defined by

$$
r_{h} \varphi \in V_{h}, \quad r_{h} \varphi\left(x_{i}\right)=\varphi\left(x_{i}\right), \quad 0 \leq i \leq m+1 \forall \varphi \in C^{0}([0,1]) .
$$

Theorem 2.1. Problem $\left(\mathrm{P}_{h}^{\tau}\right)$ has a unique solution, and we have $U_{i}^{n}>0$ for $1 \leq i \leq m+1$ and $1 \leq n \leq N$.

Proof. Assume that $U^{n}$ is known and that $U_{i}^{n}>0$ for $1 \leq i \leq m+1$; we want to find $U^{n+1}$ by using equation (2.1). We have to solve the following problem: to find $w \in V_{h}(b)$ such that $\left(\gamma(w), \varphi_{h}\right)_{h}+\tau a\left(w, \varphi_{h}\right)=\left(\gamma\left(U^{n}\right), \varphi_{h}\right)_{h} \quad \forall \varphi_{h} \in V_{h}(0)$,
where $U_{i}^{n}>0$, for $1 \leq i \leq m+1$, and $U_{0}^{n}=0$.

With the notation $w_{i}=w\left(x_{i}\right)$, equation (2.3) is equivalent to the system

$$
\begin{gathered}
\frac{h}{2} w_{i}^{2}+\frac{\tau}{h}\left(-w_{i+1}+2 w_{i}-w_{i-1}\right)=\frac{h}{2}\left(U_{i}^{n}\right)^{2} \quad \text { for } 1 \leq i \leq m, \\
w_{m+1}=b, \quad w_{0}=0 .
\end{gathered}
$$

Existence and uniqueness of a positive solution for the system (2.4) is obtained by considering a minimization problem.

Let the functional $z \rightarrow J(z)$ from $\mathbb{R}^{m}$ into $\mathbb{R}$ be defined by

$$
J(z)=\frac{1}{2} z^{t} A z+\frac{h}{6} \sum_{j=1}^{m}\left(z_{j}^{+}\right)^{3}-\sum_{j=1}^{m} F_{j} z_{j},
$$


where $F_{j}=\frac{h}{2}\left(U_{j}^{n}\right)^{2}$ for $1 \leq j \leq m-1, F_{m}=\frac{h}{2}\left(U_{m}^{n}\right)^{2}+\frac{\tau b}{h}$, and $z_{j}^{+}=$ $\max \left(z_{j}, 0\right)$. The matrix $A$ is tridiagonal, with $\frac{2 \tau}{h}$ on the diagonal and $\frac{-\tau}{h}$ on the upper and on the lower diagonal. The functional $J(\cdot)$ is strictly convex, continuous (for the Euclidean norm $\|\cdot\|_{2}$ on $\mathbb{R}^{m}$ ), and $J(z)$ tends to infinity with the Euclidean norm of the vector $z$. Then, the minimization problem:

to find $\tilde{w} \in \mathbb{R}^{m}$ such that

$$
J(\tilde{w})=\inf _{z \in \mathbb{R}^{m}} J(z)
$$

has a unique solution. The differential of the functional $J(\cdot)$ is given by

$$
J_{j}^{\prime}(z)=(A z)_{j}-F_{j}+\frac{h}{2}\left(z_{j}^{+}\right)^{2}
$$

the solution $\tilde{w}$ satisfies

$$
(A \tilde{w})_{j}-F_{j}+\frac{h}{2}\left(\tilde{w}_{j}^{+}\right)^{2}=0 \text { for } 1 \leq j \leq m
$$

Let the matrix $B$ be defined by

$$
\begin{aligned}
& B_{i i}=A_{i i}+\frac{h}{2} \tilde{w}_{i}^{+}, \\
& B_{i j}=A_{i j} \quad \text { for } i \neq j \text { and } 1 \leq i, j \leq m .
\end{aligned}
$$

Since $\left(\tilde{w}_{i}^{+}\right)^{2}=\tilde{w}_{i} \tilde{w}_{i}^{+}$, we get

$$
(B \tilde{w})_{j}=F_{j} \text { for } 1 \leq j \leq m
$$

The matrix $B$ is monotone, and the $F_{j}$ are strictly positive. We deduce that the $\tilde{w}_{j}$ are strictly positive for $1 \leq j \leq m$, so that relations (2.6) become

$$
(A \tilde{w})_{j}-F_{j}+\frac{h}{2}\left(\tilde{w}_{j}\right)^{2}=0 \text { for } 1 \leq j \leq m .
$$

From this we conclude that the problem (2.4) has at least one solution $\tilde{w}$ satisfying $\tilde{w}_{j}>0$ for $1 \leq j \leq m$.

Starting from $U^{0}$ (with $U_{j}^{0}>0$ for $1 \leq j \leq m$ ), we get the existence of $U^{n}$ by induction. To show the uniqueness of positive solutions, we assume that there exist two positive solutions $w^{1}, w^{2}$ to the problem (2.3). We have

$$
\left(\gamma\left(w^{1}\right)-\gamma\left(w^{2}\right), \varphi_{h}\right)_{h}+\tau a\left(w^{1}-w^{2}, \varphi_{h}\right)=0 \quad \forall \varphi_{h} \in V_{h}(0) .
$$

We may write

$$
\left(\gamma\left(w^{1}\right)-\gamma\left(w^{2}\right), w^{1}-w^{2}\right)_{h}=\frac{1}{2}\left(\left(w^{1}-w^{2}\right)\left(w^{1}+w^{2}\right), w^{1}-w^{2}\right)_{h} \geq 0 .
$$

Replacing $\varphi_{h}$ by $w^{1}-w^{2}$ in relation (2.9), we get $a\left(w^{1}-w^{2}, w^{1}-w^{2}\right)=0$, which yields $w^{1}=w^{2}$. Theorem 2.1 is thus proved.

Remark 2.1. If we delete the condition $U^{n} \geq 0$ in the definition of problem $\left(\mathbf{P}_{h}^{\tau}\right)$, the existence of a solution becomes an open problem. We have only an answer in the case $m=1 \quad\left(h=\frac{1}{2}\right)$. In that case, problem (2.4) becomes

$$
w_{1}^{2}+8 \tau\left(2 w_{1}-b\right)=\left(U_{1}^{n}\right)^{2}
$$

which has both positive and negative solutions.

Remark 2.2. Another proof would have been to define the function $\gamma$ by $\gamma(s)=$ $\frac{1}{2} s|s|$. In that case the problem becomes monotone, thus ensuring uniqueness and positivity. 
Lemma 2.1. The solution $\left\{U^{n}\right\}_{n=0}^{N}$ of problem $\left(\mathbf{P}_{h}^{\tau}\right)$ satisfies

$$
\begin{gathered}
\left\|U^{n}\right\|_{\infty ;(0,1)} \leq \max \left(\left\|U^{0}\right\|_{\infty ;(0,1)}, b\right) \quad \text { for } 1 \leq n \leq N, \\
\tau \sum_{n=1}^{n_{0}}\left\|U_{x}^{n}\right\|_{2 ;(0,1)}^{2} \leq C\left\|U_{x}^{0}\right\|_{2 ;(0,1)}^{2} \text { for } 1 \leq n_{0} \leq N,
\end{gathered}
$$

where the constant $C$ is independent of $h$ and $\tau$.

Proof. In relation (2.7), we replace $\tilde{w}$ by $U^{n+1}$ :

$$
\text { (2.12) } \begin{aligned}
\frac{h}{2} U_{i}^{n+1}\left(U_{i}^{n+1}\right)^{+}+\frac{\tau}{h}\left(-U_{i+1}^{n+1}+2 U_{i}^{n+1}-U_{i-1}^{n+1}\right) & =\frac{h}{2}\left(U_{i}^{n}\right)^{2} \quad \text { for } 1 \leq i \leq m, \\
U_{m+1}^{n+1}=b, \quad U_{0}^{n+1} & =0 .
\end{aligned}
$$

Let $j$ be an index such that

$$
U_{j}^{n+1}=\sup _{1 \leq i \leq m+1} U_{i}^{n+1} .
$$

If $j=m+1$, then $\sup _{1 \leq i \leq m+1} U_{i}^{n+1}=b$, and inequality (2.10) is thus proved. If $1 \leq j \leq m$, we have

$$
-U_{j+1}^{n+1}+2 U_{j}^{n+1}-U_{j-1}^{n+1} \geq 0
$$

and

$$
U_{j}^{n+1}\left(U_{j}^{n+1}\right)^{+} \leq\left(U_{j}^{n}\right)^{2} .
$$

Since the $U_{i}^{n+1}$ are strictly positive for $1 \leq i \leq m$, we get

$$
U_{j}^{n+1} \leq U_{j}^{n} \leq \sup _{1 \leq i \leq m} U_{i}^{n},
$$

from which it follows that

$$
\sup _{1 \leq i \leq m+1} U_{i}^{n+1} \leq \max \left(\sup _{1 \leq i \leq m} U_{i}^{n}, b\right)
$$

inequality (2.10) is thus proved. In relation (2.1), we replace $\varphi_{h}$ by $U^{n+1}-U^{n} \in$ $V_{h}(0)$. Summing over all indices $n, 0 \leq n \leq n_{0}-1$, we get

$$
\sum_{n=0}^{n_{0}-1}\left\{\left(\gamma\left(U^{n+1}\right)-\gamma\left(U^{n}\right), U^{n+1}-U^{n}\right)_{h}+\tau a\left(U^{n+1}, U^{n+1}-U^{n}\right)\right\}=0 .
$$

The first term of the left-hand side is positive, since the $U^{n}$ are positive for all $j$ and $n$, and

$$
\left(\gamma\left(a_{1}\right)-\gamma\left(a_{2}\right)\right)\left(a_{1}-a_{2}\right)=\frac{1}{2}\left(\left(a_{1}-a_{2}\right)^{2}\left(a_{1}+a_{2}\right)\right) \geq 0 \text { for } a_{1} \text { and } a_{2} \text { positive. }
$$

We then get

$$
\sum_{n=0}^{n_{0}-1}\left\{\left\|U_{x}^{n+1}\right\|_{2 ;(0,1)}^{2}-\left\|U_{x}^{n}\right\|_{2 ;(0,1)}^{2}+\left\|U_{x}^{n+1}-U_{x}^{n}\right\|_{2 ;(0,1)}^{2}\right\} \leq 0
$$

and

$$
\left\|U_{x}^{n_{0}}\right\|_{2 ;(0,1)}^{2} \leq\left\|U_{x}^{0}\right\|_{2 ;(0,1)}^{2} .
$$

From the last inequality we deduce inequality (2.11). Lemma 2.1 is thus proved. 


\section{ERROR BOUNDS}

A variational technique, first developed by Nochetto [16] for the Stefan problem, and based on integration by parts, is used in this section. We also mention two relevant works of Nochetto and Verdi [17] and Elliott [10], concerning the effect of numerical integration in multidimensional degenerate parabolic problems.

We begin this section with recalling a numerical integration result. This result will be used in the proof of the error estimate, because we use the lumped mass version of the $L^{2}$ scalar product for the scheme (2.1). We define the quantity $E_{h}$ by

We have:

$$
E_{h}(v, w)=(v, w)_{h}-\int_{0}^{1} v w d x \quad \forall v, w \in C^{0}([0,1])
$$

Lemma 3.1. The following estimate is valid for any $p \geq 2$ :

$$
\begin{aligned}
\left|E_{h}\left(v^{p}, w\right)\right| \leq C h^{2}\left\{\left\|v^{p-1}\right\|_{L^{\infty}(0,1)}\left\|v_{x}\right\|_{L^{2}(0,1)}\left\|w_{x}\right\|_{L^{2}(0,1)}\right. & \\
& \left.+\left\|v^{p-2}\right\|_{L^{\infty}(0,1)}\left\|v_{x}\right\|_{L^{2}(0,1)}^{2}\|w\|_{L^{\infty}(0,1)}\right\} \quad \forall v, w \in V_{h}(b) .
\end{aligned}
$$

Proof. The classical tools of the proof are developed, for example, in Ciarlet [7]. For a detailed proof, we refer to Lesaint and Pousin [15].

We introduce the following interpolation operators:

$$
\begin{aligned}
\Pi_{h}: V(b) \rightarrow V_{h}(b) & \\
v & \mapsto \Pi_{h} v \quad \text { defined by } a\left(v-\Pi_{h} v, \varphi\right)=0 \forall \varphi \in V_{h}(0), \\
\Pi_{h}^{\tau}: L^{2}(0, T ; V(b)) & \rightarrow W_{h}^{\tau}(b) \\
w & \mapsto \Pi_{h}^{\tau} w \quad \text { defined by }\left.\Pi_{h}^{\tau} w\right|_{\left(t_{n}, t_{n+1}\right]}=\frac{1}{\tau} \int_{t_{n}}^{t_{n+1}} \Pi_{h}(\cdot, t) d t,
\end{aligned}
$$

where $V(b)$ is the following space:

$$
V(b)=\left\{v \in H^{1}(0,1) ; v(0)=0, v(1)=b\right\} .
$$

We let $Y=\Pi_{h}^{\tau} u$, where $u$ is the exact solution of problem $(\mathrm{P})$. For any $w \in$ $C^{0}\left(\overline{Q_{T}}\right)$, we define $q^{\tau} w \in L^{2}\left(0, T ; C^{0}([0,1])\right)$ by

$$
\begin{aligned}
\left.q^{\tau} w\right|_{\left(t_{n}, t_{n+1}\right]} & =w\left(\cdot, t_{n+1}\right)=w^{n+1}(\cdot) \quad \text { for } 0 \leq n \leq N-1, \\
\left(q^{\tau} w\right)(\cdot, 0) & =w(\cdot, 0)=w^{0} .
\end{aligned}
$$

Finally, we define $U \in W_{h}^{\tau}(b)$ by

$$
\left.U\right|_{\left(t_{n}, t_{n+1}\right]}=U^{n+1},
$$

where $U^{n+1} \in V_{h}(b)$ is defined by the scheme (2.1).

Before stating the theorem for the error estimate, we show how a stability inequality in $L^{2}$ norm can be established for the continuous version of the problem; this will give us a better understanding of the rather long proof of this stability inequality for the discrete problem.

In the next few lines, we assume that $\tilde{u}$ is a smooth function and that $b=0$. A continuous version of scheme (2.1) may be written as follows:

$$
\left(\gamma(\tilde{u})_{t}, \varphi\right)+a(\tilde{u}, \varphi)=0 \quad \forall \varphi \text { smooth, } \varphi(0, t)=\varphi(1, t)=0 .
$$


We now define the function $\varphi$ by

$$
\varphi(x, s)= \begin{cases}0 & \text { for } t \leq s \leq T, \\ v(x, s)=\int_{s}^{t} \tilde{u}(x, \sigma) d \sigma & \text { for } 0 \leq s \leq t .\end{cases}
$$

Integrating equation (3.1) with respect to time, we get

$$
\int_{0}^{t}\left(\frac{\partial}{\partial s} \gamma(\tilde{u}), \int_{s}^{t} \tilde{u} d \sigma\right) d s+\int_{0}^{t} a\left(\tilde{u}, \int_{s}^{t} \tilde{u} d \sigma\right) d s=0 .
$$

By integration by parts, the first term of the left-hand side of relation (3.2) may be written as

$$
\begin{aligned}
& \left.\left(\gamma(\tilde{u}), \int_{s}^{t} \tilde{u} d \sigma\right)\right|_{0} ^{t}+\int_{0}^{t}(\gamma(\tilde{u}), \tilde{u}) d s \\
& =-\left(\gamma(\tilde{u}(\cdot, 0)), \int_{0}^{t} \tilde{u} d \sigma\right)+\int_{0}^{t} \int_{0}^{1} \frac{\tilde{u}^{3}}{2} d x d s .
\end{aligned}
$$

Since $\frac{\partial v}{\partial s}=-\tilde{u}$, the second term of the left-hand side of relation (3.2) may be written as

$$
\int_{0}^{t}-a\left(\frac{\partial v}{\partial s}, v\right) d s=\int_{0}^{t} \int_{0}^{1}-\frac{\partial^{2} v}{\partial x \partial s} \frac{\partial v}{\partial x} d x d s=\frac{1}{2} \int_{0}^{1}\left(\frac{\partial v}{\partial x}\right)^{2}(x, 0) d x,
$$

since $v(x, t)=0$. Multiplying relation (3.2) by 2 , we deduce

$$
\begin{gathered}
\int_{0}^{t} \int_{0}^{1} \tilde{u}^{3} d x d s+a\left(\int_{0}^{t} \tilde{u}(\cdot, s) d s, \int_{0}^{t} \tilde{u}(\cdot, s) d s\right) \\
=\int_{0}^{1}\left(\tilde{u}_{0}^{2}(x) \int_{0}^{t} \tilde{u}(x, s) d s\right) d x .
\end{gathered}
$$

Poincaré's inequality reads here as follows:

$$
\|\varphi\|_{2 ;(0,1)}^{2} \leq a(\varphi, \varphi) \quad \forall \varphi \text { such that } \varphi(0, t)=0 .
$$

Therefore,

$$
\int_{0}^{1}\left(\int_{0}^{t} \tilde{u}(x, s) d s\right)^{2} d x \leq a\left(\int_{0}^{t} \tilde{u}(x, s) d s, \int_{0}^{t} \tilde{u}(x, s) d s\right) .
$$

The right-hand side of relation (3.3) is controlled using the Schwarz inequality, so we get

$$
\int_{0}^{t} \int_{0}^{1} \tilde{u}^{3} d x d s+\frac{1}{2} a\left(\int_{0}^{t} \tilde{u}(\cdot, s) d s, \int_{0}^{t} \tilde{u}(\cdot, s) d s\right) \leq \frac{1}{2} \int_{0}^{1} \tilde{u}_{0}^{4}(x) d x .
$$

Using the property $\tilde{u}(x, t) \geq \beta x$ for $0 \leq t<T$ and for $0 \leq x \leq 1$, we deduce that for all $z$ such that $0<z<1$ we have

$$
\int_{0}^{1} \tilde{u}^{3} d x \geq \int_{z}^{1} \tilde{u}^{3} d x \geq \beta z \int_{z}^{1} \tilde{u}^{2} d x=\beta z \int_{0}^{1} \tilde{u}^{2} d x-\beta z \int_{0}^{z} \tilde{u}^{2} d x .
$$

Combining this inequality with inequality (3.4), we get the following stability estimate in $L^{2}$ norm:

$$
\begin{aligned}
& \beta z \int_{0}^{1} \int_{0}^{t} \tilde{u}^{2}(x, s) d s d x+\frac{1}{2} a\left(\int_{0}^{t} \tilde{u}(\cdot, s) d s, \int_{0}^{t} \tilde{u}(\cdot, s) d s\right) \\
& \quad \leq \frac{1}{2} \int_{0}^{1} \tilde{u}_{0}^{4}(x) d x+\beta z \int_{0}^{t} \int_{0}^{z} \tilde{u}^{2}(x, s) d x d s .
\end{aligned}
$$


Theorem 3.1. Let $u$ be the solution of problem $(\mathrm{P})$, and let $U \in W_{h}^{\tau}(b)$ be the solution of problem $\left(\mathbf{P}_{h}^{\tau}\right)$. Then

$$
\|U-u\|_{L^{2}\left(Q_{T}\right)} \leq C\left(\frac{\tau}{h}+h^{1 / 2}\right),
$$

where the constant $C$ is independent of $\tau$ and $h$.

Proof. First, we give a stability inequality in $L^{2}$ norm for the problem $\left(\mathrm{P}_{h}^{\tau}\right)$, analogous to inequality (3.5). This inequality allows us to control the difference between the approximate solution and some $W_{h}^{\tau}(b)$ interpolate of the exact solution.

For $0 \leq n \leq N-1$, we may write

$$
\begin{aligned}
\int_{t_{n}}^{t_{n+1}}\left(\gamma(u)_{t}, \varphi\right) d t+\int_{t_{n}}^{t_{n+1}} a(u, \varphi) d t & =0 \\
\forall \varphi & \in V_{h}(0), \gamma(u)(x, 0)=\gamma\left(u_{0}\right)(x) .
\end{aligned}
$$

Using the notations introduced above, and summing over the indices from $n=$ 0 to $n=n_{0}-1$, we get

$$
\begin{aligned}
& \sum_{n=0}^{n_{0}-1}\left(\gamma\left(u^{n+1}\right)-\gamma\left(u^{n}\right), \varphi^{n+1}\right)+\tau \sum_{n=0}^{n_{0}-1} a\left(Y^{n+1}, \varphi^{n+1}\right) \\
& \forall \varphi \in W_{h}^{\tau}(0), 1 \leq n_{0} \leq N, \quad \gamma\left(u^{0}\right)=\gamma\left(u_{0}\right) .
\end{aligned}
$$

Summing the relations (2.1) over the indices from $n=0$ to $n_{0}-1$, we get

$$
\begin{aligned}
& \sum_{n=0}^{n_{0}-1}\left(\gamma\left(U^{n+1}\right)-\gamma\left(U^{n}\right), \varphi^{n+1}\right)_{h}+\tau \sum_{n=0}^{n_{0}-1} a\left(U^{n+1}, \varphi^{n+1}\right)=0 \\
& \forall \varphi \in W_{h}^{\tau}(0), 1 \leq n_{0} \leq N, \gamma\left(U^{0}\right)=\gamma\left(r_{h} u_{0}\right) .
\end{aligned}
$$

In relations (3.8) and (3.9), we integrate by parts, in a discrete way with respect to time. We get

$$
\begin{gathered}
-\sum_{n=1}^{n_{0}}\left(\gamma\left(u^{n}\right), \varphi^{n+1}-\varphi^{n}\right)+\tau \sum_{n=1}^{n_{0}} a\left(Y^{n}, \varphi^{n}\right)-\left(\gamma\left(u^{0}\right), \varphi^{1}\right)+\left(\gamma\left(u^{n_{0}}\right), \varphi^{n_{0}+1}\right)=0, \\
-\sum_{n=1}^{n_{0}}\left(\gamma\left(U^{n}\right), \varphi^{n+1}-\varphi^{n}\right)_{h}+\tau \sum_{n=1}^{n_{0}} a\left(U^{n}, \varphi^{n}\right)-\left(\gamma\left(U^{0}\right), \varphi^{1}\right)_{h} \\
+\left(\gamma\left(U^{n_{0}}\right), \varphi^{n_{0}+1}\right)_{h}=0 .
\end{gathered}
$$

Subtracting these equalities yields

$$
\begin{aligned}
& \sum_{n=1}^{n_{0}}\left[-\left(\gamma\left(U^{n}\right)-\gamma\left(u^{n}\right), \varphi^{n+1}-\varphi^{n}\right)+\tau a\left(U^{n}-Y^{n}, \varphi^{n}\right)\right] \\
&=-\left(\gamma\left(U^{0}\right)-\gamma\left(u^{0}\right), \varphi^{1}\right) \\
&+\sum_{n=1}^{n_{0}} E_{h}\left(\gamma\left(U^{n}\right), \varphi^{n+1}-\varphi^{n}\right)-E_{h}\left(\gamma\left(U^{0}\right), \varphi^{1}\right) \\
&-\left(\gamma\left(U^{n_{0}}\right), \varphi^{n_{0}+1}\right)_{h}+\left(\gamma\left(u^{n_{0}}\right), \varphi^{n_{0}+1}\right) \\
& \forall \varphi \in W_{h}^{\tau}(0), 1 \leq n_{0} \leq N .
\end{aligned}
$$


Let $\varphi^{n}$ be the following discrete integral:

$$
\varphi^{n}= \begin{cases}\tau \sum_{k=n}^{n_{0}}\left(U^{k}-Y^{k}\right) & \text { if } 1 \leq n \leq n_{0}, \\ 0 & \text { if } n_{0}+1 \leq n \leq N .\end{cases}
$$

It is easy to see that $\varphi^{n} \in V_{h}(0), \varphi^{n+1}-\varphi^{n}=-\tau\left(U^{n}-Y^{n}\right)$. Replacing $\varphi^{n}$ by its value in equality $(3.10)$, we have

$$
\begin{aligned}
\tau \sum_{n=1}^{n_{0}}\left(\gamma\left(U^{n}\right)-\gamma\left(u^{n}\right), U^{n}-Y^{n}\right)+\tau^{2} \sum_{n=1}^{n_{0}} a\left(U^{n}-Y^{n}, \sum_{k=n}^{n_{0}}\left(U^{k}-Y^{k}\right)\right) \\
=-\tau \sum_{n=1}^{n_{0}} E_{h}\left(\gamma\left(U^{n}\right), U^{n}-Y^{n}\right)-\tau \sum_{k=1}^{n_{0}} E_{h}\left(\gamma\left(U^{0}\right), U^{k}-Y^{k}\right) \\
-\tau \sum_{k=1}^{n_{0}}\left(\gamma\left(U^{0}\right)-\gamma\left(u^{0}\right), U^{k}-Y^{k}\right) .
\end{aligned}
$$

This equality may be written, with obvious notations,

$$
D_{1}+D_{2}=D_{3}+D_{4}+D_{5}
$$

First, we estimate the term $D_{1}$. To do that, we rewrite $D_{1}$ as the sum of two terms:

$$
\begin{aligned}
D_{1}=\overline{D_{1}}+\widetilde{D_{1}}= & \tau \sum_{n=1}^{n_{0}}\left(\gamma\left(U^{n}\right)-\gamma\left(u^{n}\right), U^{n}-u^{n}\right) \\
& +\tau \sum_{n=1}^{n_{0}}\left(\gamma\left(U^{n}\right)-\gamma\left(u^{n}\right), u^{n}-Y^{n}\right)
\end{aligned}
$$

We have

$$
\overline{D_{1}}=\frac{\tau}{2} \sum_{n=1}^{n_{0}} \int_{0}^{1}\left(U^{n}+u^{n}\right)\left(U^{n}-u^{n}\right)^{2} d x
$$

with $U^{n} \geq 0$. The inequality $u(x, t) \geq \beta x$ for $0 \leq t \leq T, 0 \leq x \leq 1$ (see Theorem 1.1) implies $u^{n}(x) \geq \beta h$ for $h \leq x, 1 \leq n \leq N$. Hence,

$\int_{0}^{1}\left(U^{n}+u^{n}\right)\left(U^{n}-u^{n}\right)^{2} d x \geq \int_{h}^{1}\left(U^{n}+u^{n}\right)\left(U^{n}-u^{n}\right)^{2} d x \geq \beta h \int_{h}^{1}\left(U^{n}-u^{n}\right)^{2} d x$,

which leads to the inequality

$$
\begin{aligned}
\frac{\beta h}{2} \tau \sum_{n=1}^{n_{0}} \int_{0}^{1}\left(U^{n}-u^{n}\right)^{2} d x & \leq \overline{D_{1}}+\frac{\beta h}{2} \tau \sum_{n=1}^{n_{0}} \int_{0}^{h}\left(U^{n}-u^{n}\right)^{2} d x \\
& \leq \overline{D_{1}}+\beta h^{2} \tau \sum_{n=1}^{n_{0}}\left(\left\|U^{n}\right\|_{\infty ;(0,1)}^{2}+\left\|u^{n}\right\|_{\infty ;(0,1)}^{2}\right) .
\end{aligned}
$$

Using Theorem 1.1 and Lemma 2.1, we get

$$
\frac{\beta h}{2}\left\|U-q^{\tau} u\right\|_{L^{2}\left(\overline{Q_{T_{0}}}\right)}^{2} \leq \overline{D_{1}}+\mathscr{C}_{1} h^{2}
$$

where $\mathscr{C}_{1}$ is a constant independent of $h$. 
To get an estimate of $\widetilde{D_{1}}$, we let

$$
a_{n}= \begin{cases}\left(\gamma\left(U^{n}\right)-\gamma\left(u^{n}\right)\right) /\left(U^{n}-u^{n}\right) & \text { if } U^{n} \neq u^{n}, \\ 0 & \text { if } U^{n}=u^{n} .\end{cases}
$$

This expression is uniformly bounded in $L^{\infty}\left(Q_{T}\right)$. We write

$$
\begin{aligned}
& \left(\gamma\left(U^{n}\right)-\gamma\left(u^{n}\right), u^{n}-Y^{n}\right) \\
& \quad=\left(\left(\left(\gamma\left(U^{n}\right)-\gamma\left(u^{n}\right)\right)\left[U^{n}-u^{n}\right]\right)^{1 / 2}, a_{n}^{1 / 2}\left(u^{n}-Y^{n}\right)\right) .
\end{aligned}
$$

Using Schwarz's inequality, we get

$$
\begin{aligned}
\left|\widetilde{D_{1}}\right| \leq & {\left[\tau \sum_{n=1}^{n_{0}}\left(\gamma\left(U^{n}\right)-\gamma\left(u^{n}\right), U^{n}-u^{n}\right)\right]^{1 / 2} } \\
& \times\left[\tau \sum_{n=1}^{n_{0}}\left\|a_{n}\right\|_{\infty ; Q_{T}}\left(u^{n}-Y^{n}, u^{n}-Y^{n}\right)\right]^{1 / 2} .
\end{aligned}
$$

Letting $A=\max _{1 \leq n \leq N}\left(\left\|a_{n}\right\|_{\infty} ; Q_{T}\right)$, we get

$$
\left|\widetilde{D_{1}}\right| \leq \varepsilon \overline{D_{1}}+\frac{A}{4 \varepsilon}\left\|q^{\tau} u-Y\right\|_{2}^{2} ; Q_{T_{0}}
$$

for any $\varepsilon>0$ with $T_{0}=n_{0} \tau$. Since $D_{1} \geq \overline{D_{1}}-\left|\widetilde{D_{1}}\right|$, we have

$$
D_{1} \geq \overline{D_{1}}(1-\varepsilon)-\frac{A}{4 \varepsilon}\left\|q^{\tau} u-Y\right\|_{2}^{2} ; Q_{T_{0}} .
$$

Choosing $\varepsilon=\frac{1}{2}$ and using inequality (3.13), we finally get

$$
\frac{\beta h}{4}\left\|U-q^{\tau} u\right\|_{2}^{2}, Q_{T_{0}}-\frac{A}{2}\left\|q^{\tau} u-Y\right\|_{2}^{2}, Q_{T_{0}} \leq D_{1}+\frac{\mathscr{C}_{1} h^{2}}{2} .
$$

Consider the term

$$
D_{2}=\tau^{2} \sum_{n=1}^{n_{0}} a\left(U^{n}-Y^{n}, \sum_{k=n}^{n_{0}}\left(U^{k}-Y^{k}\right)\right),
$$

and let $b_{n}=\left(U^{n}-Y^{n}\right)_{x}$; we have

$$
D_{2}=\tau^{2} \sum_{n=1}^{n_{0}} \int_{0}^{1} b_{n} \sum_{k=n}^{n_{0}} b_{k} d x .
$$

We easily check that

$$
\sum_{n=1}^{n_{0}}\left(b_{n} \sum_{k=n}^{n_{0}} b_{k}\right) \geq \frac{1}{2} \sum_{k=1}^{n_{0}} b_{k}^{2}
$$

and we deduce

$$
D_{2} \geq \frac{1}{2}\left\|\tau \sum_{k=1}^{n_{0}}\left(U^{k}-Y^{k}\right)_{x}\right\|_{2 ;(0,1)}^{2}
$$

Consider the term

$$
D_{3}=-\tau \sum_{n=1}^{n_{0}} E_{h}\left(\gamma\left(U^{n}\right), U^{n}-Y^{n}\right) .
$$


Using Lemma 3.1, with $p=2$, we have

$$
\begin{aligned}
& \left|E_{h}\left(\gamma\left(U^{n}\right), U^{n}-Y^{n}\right)\right| \\
& \quad \leq C h^{2}\left\{\left\|U^{n}\right\|_{\infty ;(0,1)}\left\|U_{x}^{n}\right\|_{2 ;(0,1)}\left\|\left(U^{n}-Y^{n}\right)_{x}\right\|_{2 ;(0,1)}\right. \\
& \left.\quad+\left\|U_{x}^{n}\right\|_{2 ;(0,1)}^{2}\left\|U^{n}-Y^{n}\right\|_{\infty ;(0,1)}\right\} .
\end{aligned}
$$

Lemma 2.1 implies $\left\|U^{n}\right\|_{\infty ;(0,1)} \leq C$. Since

$$
u \in C^{0}\left(\overline{Q_{T}}\right) \cap L^{\infty}\left(0, T ; W^{1, \infty}(0,1)\right),
$$

we derive from the definition of $Y$ that

$$
\left\|Y^{n}\right\|_{\infty ;(0,1)} \leq C \text {. }
$$

The right-hand side of inequality (3.17) is thus bounded by

$$
\begin{gathered}
C h^{2}\left(\left\|U_{x}^{n}\right\|_{2 ;(0,1)}\left\|\left(U^{n}-Y^{n}\right)_{x}\right\|_{2 ;(0,1)}+\left\|U_{x}^{n}\right\|_{2 ;(0,1)}^{2}\right) \\
\leq 3 C h^{2}\left\{\left\|U_{x}^{n}\right\|_{2 ;(0,1)}^{2}+\left\|Y_{x}^{n}\right\|_{2 ;(0,1)}^{2}\right\}
\end{gathered}
$$

where $C$ denotes various constants independent of $h$. Using once more Lemma 2.1 (inequality (2.11)) and the definition of $Y^{n}$, we get

$$
\left|D_{3}\right| \leq \mathscr{C}_{2} h^{2}
$$

where $\mathscr{C}_{2}$ depends on $\left\|U^{0}\right\|_{\infty ;(0,1)},\left\|U_{x}^{0}\right\|_{\infty ;(0,1)},\|u\|_{L^{2}\left(0, T ; H^{1}(0,1)\right)}$, and $b$. We proceed in the same way to estimate the term

$$
D_{4}=-\tau \sum_{k=1}^{n_{0}} E_{h}\left(\gamma\left(U^{0}\right), U^{k}-Y^{k}\right)
$$

and we get

$$
\left|D_{4}\right| \leq \mathscr{C}_{3} h^{2}
$$

where $\mathscr{C}_{3}$ is a constant of the same kind as $\mathscr{C}_{2}$.

For the last term,

$$
D_{5}=-\tau \sum_{k=1}^{n_{0}}\left(\gamma\left(U^{0}\right)-\gamma\left(u^{0}\right), U^{k}-Y^{k}\right),
$$

we use Schwarz's inequality to get

$$
\left|D_{5}\right| \leq \frac{\tau}{2}\left\|U^{0}+u^{0}\right\|_{\infty ;(0,1)}\left\|U^{0}-u^{0}\right\|_{2 ;(0,1)}\left\|\sum_{k=1}^{n_{0}} U^{k}-Y^{k}\right\|_{2 ;(0,1)} .
$$

Since $u^{0} \in W^{1, \infty}(0,1)$, we easily check that

$$
\left\|U^{0}-u^{0}\right\|_{2 ;(0,1)} \leq C h ; \quad\left\|U^{0}+u^{0}\right\|_{\infty ;(0,1)} \leq C,
$$

where $C$ denotes different constants independent of $h$. Since $\left(U^{k}-Y^{k}\right)(0)=$ 0 , for all $h$, we may use Poincaré's inequality, and we get

$$
\left|D_{5}\right| \leq \mathscr{C}_{4} h\left\|\tau \sum_{k=1}^{n_{0}} U_{x}^{k}-Y_{x}^{k}\right\|_{2 ;(0,1)} .
$$


Combining relations (3.12) and (3.15)-(3.20), we get

$$
\begin{gathered}
\frac{\beta h}{4}\left\|U-q^{\tau} u\right\|_{2 ; Q_{T_{0}}}^{2}-\frac{A}{2}\left\|q^{\tau} u-Y\right\|_{2 ; Q_{T_{0}}}^{2}-\frac{C h^{2}}{2}+\frac{1}{2}\left\|\tau \sum_{k=1}^{n_{0}} U_{x}^{k}-Y_{x}^{k}\right\|_{2 ;(0,1)}^{2} \\
\leq\left(\mathscr{C}_{2}+\mathscr{C}_{3}\right) h^{2}+\mathscr{C}_{4} h\left\|\tau \sum_{k=1}^{n_{0}} U_{x}^{k}-Y_{x}^{k}\right\|_{2 ;(0,1)} .
\end{gathered}
$$

From this inequality we deduce a stability estimate in $L^{2}$ norm:

$$
\begin{aligned}
\beta h\left\|U-q^{\tau} u\right\|_{2 ; Q_{0}}^{2}+\left\|\tau \sum_{k=1}^{n_{0}} U_{x}^{k}-Y_{x}^{k}\right\|_{2 ;(0,1)}^{2} \\
\leq\left(2 \mathscr{C}_{1}+4 \mathscr{C}_{2}+4 \mathscr{C}_{3}+4 \mathscr{C}_{4}\right) h^{2}+2 A\left\|q^{\tau} u-Y\right\|_{2 ; Q_{T_{0}}}^{2}
\end{aligned}
$$

It remains to estimate the term $q^{\tau} u-Y$. We write

We have

$$
\begin{aligned}
q^{\tau} u-\left.Y\right|_{\left(t_{n}, t_{n+1}\right]}= & \frac{1}{\tau} \int_{t_{n}}^{t_{n+1}}\left(u\left(\cdot, t_{n+1}\right)-\Pi_{h} u(\cdot, t)\right) d t \\
= & \frac{1}{\tau} \int_{t_{n}}^{t_{n+1}}\left(u\left(\cdot, t_{n+1}\right)-u(\cdot, t)\right) d t \\
& +\frac{1}{\tau} \int_{t_{n}}^{t_{n+1}}\left(u(\cdot, t)-\Pi_{h} u(\cdot, t)\right) d t \\
= & E_{1}^{n}+E_{2}^{n} .
\end{aligned}
$$

$$
\begin{aligned}
\left\|E_{1}^{n}\right\|_{2 ;(0,1)}^{2}= & \frac{1}{\tau^{2}} \int_{0}^{1}\left(\int_{t_{n}}^{t_{n+1}} u\left(x, t_{n+1}\right)-u\left(x, t_{n}\right) d t\right)^{2} d x \\
= & \frac{1}{\tau^{2}} \int_{h}^{1}\left(\int_{t_{n}}^{t_{n+1}} u\left(x, t_{n+1}\right)-u\left(x, t_{n}\right) d t\right)^{2} d x \\
& +\frac{1}{\tau^{2}} \int_{0}^{h}\left(\int_{t_{n}}^{t_{n+1}} u\left(x, t_{n+1}\right)-u\left(x, t_{n}\right) d t\right)^{2} d x
\end{aligned}
$$

For the first term of the right-hand side, we may write

$$
\begin{aligned}
& \int_{h}^{1}\left(\int_{t_{n}}^{t_{n+1}} u\left(x, t_{n+1}\right)-u\left(x, t_{n}\right) d t\right)^{2} d x \\
& \quad=\int_{h}^{1}\left(\int_{t_{n}}^{t_{n+1}} \int_{t_{n}}^{t_{n+1}} u_{t}(x, s) d s d t\right)^{2} d x \\
& \leq \tau^{3} \int_{h}^{1} \int_{t_{n}}^{t_{n+1}} u_{t}^{2}(x, t) d t d x .
\end{aligned}
$$

For the second term of the right-hand side, we use property (i) of Theorem 1.1 to get

$$
0 \leq u(x, t) \leq C h \text { for any } 0 \leq x \leq h, 0 \leq t \leq T
$$

and we obtain the estimate

$$
\int_{0}^{h}\left(\int_{t_{n}}^{t_{n+1}} u\left(x, t_{n+1}\right)-u\left(x, t_{n}\right) d t\right)^{2} d x \leq C \tau^{2} h^{3}
$$


From inequalities (3.22) and (3.23) we get, by using property (ii) of Theorem 1.1 with $z=h$,

$$
\tau \sum_{n=1}^{n_{0}}\left\|E_{1}^{n}\right\|_{2 ;(0,1)}^{2} \leq C\left(\frac{\tau^{2}}{h}+h^{3}\right) .
$$

On the other hand, it is well known that

$$
\left\|u(\cdot, t)-\Pi_{h} u(\cdot, t)\right\|_{2 ;(0,1)}^{2} \leq C h^{2}\|u\|_{L^{\infty}\left(0, T ; H^{1}(0,1)\right)}^{2} ;
$$

thus we deduce

$$
\left\|q^{\tau} u-Y\right\|_{2 ; Q_{T_{0}}}^{2} \leq \mathscr{C}_{5}\left(\frac{\tau^{2}}{h}+h^{2}\right) .
$$

Combining inequality (3.21) and (3.24), we get

$$
\left\|U-q^{\tau} u\right\|_{2 ; Q_{T_{0}}}^{2} \leq \mathscr{C}_{6}\left(\frac{\tau^{2}}{h^{2}}+h\right)
$$

for any $T_{0}=n_{0} \tau, n_{0} \leq N$.

In the same way as before, we obtain

$$
\left\|u-q^{\tau} u\right\|_{2, Q_{T}}^{2} \leq C\left(\frac{\tau^{2}}{h}+h^{3}\right) .
$$

Finally, we get, by using the triangular inequality,

$$
\|U-u\|_{2 ; Q_{T}} \leq \mathscr{C}_{7}\left(\frac{\tau}{h}+h^{1 / 2}\right),
$$

so that Theorem 3.1 is proved.

\section{AN algorithm to calculate the solution of problem $\left(\mathbf{P}_{h}^{\tau}\right)$}

The definition of the approximate problem (equation (2.1)) implies that for each time step, we have to solve a problem of the form:

for a given function $f \in C^{0}([0,1]), f>0$ in $(0,1]$, find $w \in V_{h}(b), w>0$ in $(0,1]$, such that

$$
a(w, v)+\left(\frac{1}{2} w^{2}, v\right)_{h}=(f, v)_{h} \quad \forall v \in V_{h}(0) .
$$

Consider the following algorithm:

let $w_{0} \in V_{h}(b)$ be given; define the sequence $\left\{w_{k}\right\}, w_{k} \in$ $V_{h}(b)$, by

$$
a\left(w_{k+1}, v\right)+\frac{1}{2}\left(w_{k+1} w_{k}, v\right)_{h}=(f, v)_{h} \quad \forall v \in V_{h}(0) .
$$

The proof of Theorem 2.1 shows that, for a given $w^{k} \in V_{h}(b)$ with $w^{k}>0$ for $x>0$, the problem (4.2) has a unique solution $w^{k+1} \in V_{h}(b)$ satisfying $w^{k+1}>0$ for $x>0$.

Before stating the convergence result for the algorithm (4.2), we introduce some notations, and we give some intermediate results, which will be proved at the end of this section. 
Let $H_{b}$ be the space $V_{h}(b)$ equipped with the maximum norm. Define the mapping

$$
\begin{aligned}
S_{f}^{b}: H_{b} & \rightarrow H_{b} \\
\varphi & \mapsto \theta=S_{f}^{b}(\varphi),
\end{aligned}
$$

where $\theta \in V_{h}(b)$ is a solution of

$$
a(\theta, v)+\frac{1}{2}(\varphi \theta, v)_{h}=(f, v)_{h} \quad \forall v \in V_{h}(0) .
$$

Then the following inclusion holds true.

Lemma 4.1. The domain $\mathscr{D}\left(S_{f}^{b}\right)$ of the operator $S_{f}^{b}$ satisfies $\mathscr{D}\left(S_{f}^{b}\right) \supset\{\varphi \in$ $\left.H_{b} ; \inf \varphi \geq 0\right\}$.

It is easy to check that $w_{k+1}$ can be expressed as

$$
w_{k+1}=S_{f}^{b}\left(w_{k}\right)
$$

and that $w$, the solution of problem (4.1), satisfies

$$
w=S_{f}^{b}(w) .
$$

Moreover, we have $w_{j}=w\left(x_{j}\right)>0$ for $1 \leq j \leq m+1$.

Now, we are interested in the existence of the derivative of $S_{f}^{b}$. Let $\bar{w}$ be defined by

$$
\bar{w}=\inf _{j \geq 1} w_{j}
$$

then we have the following result.

Lemma 4.2. Let $\Gamma$ be the neighborhood of $w$ in $H_{b}$ defined by

$$
\Gamma=\left\{\varphi \in H_{b} ;\|w-\varphi\|_{\infty ;(0,1)}<\bar{w} / 2\right\} .
$$

Then $S_{f}^{b}(\varphi) \in C^{1}(\Gamma)$, and the differential $D S_{f}^{b}$ of $S_{f}^{b}$ is given by

$$
D S_{f}^{b}(\varphi) \cdot v=-S(\varphi) \cdot v S_{f}^{b}(\varphi),
$$

where the linear operator $y \mapsto \psi=S(\varphi) \cdot y: H_{b} \rightarrow V_{h}(0)$ is defined by

$$
a(\psi, v)+\frac{1}{2}(\varphi \psi, v)_{h}=\frac{1}{2}(y, v)_{h} \quad \forall v \in V_{h}(0) .
$$

The next lemma provides an estimate of the spectral radius of the mapping $D S_{f}^{b}(w)$.

Lemma 4.3. The spectral radius of the mapping $v \rightarrow D S_{f}^{b}(w) \cdot v$ from $H_{0}$ into $H_{0}$ satisfies

$$
\rho\left(D S_{f}^{b}(w)\right) \leq \frac{\|w\|_{\infty ;(0,1)}}{2 \mu+\|w\|_{\infty ;(0,1)}}
$$

where

$$
\mu=\inf _{q \in V_{h}(0), q \neq 0} \frac{a(q, q)}{(q, q)_{h}}>0 .
$$

We are now in a position to show that the algorithm (4.2) is convergent in the following sense. 
Theorem 4.1. Let $w$ be the solution of problem (4.1). There exists a neighborhood of $w$ in $L^{\infty}(0,1)$, denoted by $\Gamma_{1}$, such that if $w_{0}$ is chosen in $\Gamma_{1}$, the sequence $\left\{w^{k}\right\}$ converges towards $w$.

Proof. We want to show that for a certain norm on the (finite-dimensional) space $V_{h}(b)$, the mapping $S_{f}^{b}$ is locally a contraction. To do that, we show that the derivative of $S_{f}^{b}$ is bounded by a constant $<1$ in a neighborhood of $w$.

It is well known (see, for example, Ciarlet [6, p. 19]) that for any $\varepsilon$, there exists a norm $\||\cdot|||$ on $V_{h}(0)$ such that for the induced norm for the operators, we have

$$
\left\|\left|D S_{f}^{b}(w) \|\right| \leq \rho\left(D S_{f}^{b}(w)\right)+\varepsilon .\right.
$$

Choosing $\varepsilon$ small enough, by Lemma 4.3 we get the existence of

$$
k \in \mathbb{R} \text { such that }\left\|\left|D S_{f}^{b}(w)\right|\right\| \leq k<1 .
$$

Applying Lemma 4.2, we get the existence of a neighborhood $\Gamma_{2}(w) \subset \Gamma$ and a real number $k^{\prime}$ such that

$$
\left\|D S_{f}^{b}(\varphi)\right\| \leq k^{\prime}<1 \quad \forall \varphi \in \Gamma_{2}(w) .
$$

Let $\beta$ be the maximum of radius $R$ such that $B_{R}(w) \subset \Gamma_{2}(w)$, where $B_{R}(w)$ is the ball of center $w$ and radius $R$, for the norm $\|\cdot \cdot\| \mid$. Set $\Gamma_{1}(w)=B_{\beta}(w)$. Then, it is not difficult to check that a classical fixed point theorem applies (see, for example, Dieudonné [8, p. 261]), so Theorem 4.1 is proved.

Remark 4.1. For the approximate problem (2.1) we have to consider the problem $(4.1)$ where the term $\left(\frac{1}{2} w^{2}, v\right)_{h}$ is replaced by $\left(\frac{1}{2 \tau} w^{2}, v\right)_{h}$. In fact, Theorem 4.1 still holds if the term $\left(\frac{1}{2} w^{2}, v\right)_{h}$ is replaced by $\left(\alpha w^{2}, v\right)_{h}$ for any $\alpha>0$. We just have to modify the definition of $\mu$. Nevertheless, let us mention that for the approximate problem (2.1), when $h$ is fixed, $\mu$ depends linearly on $\tau$. Consequently, the speed of convergence decreases with $\tau$, since the spectral radius of the mapping $D S_{f}^{b}(w)$ goes to one when $\tau$ tends to zero.

To conclude this section, we give the proofs of Lemmas 4.2 and 4.3.

Proof of Lemma 4.2. For $\varphi \in \Gamma$, let $\tilde{\theta}$ and $\theta$ be the functions of $V_{h}(b)$ respectively defined by

$$
\begin{gathered}
a(\tilde{\theta}, \psi)+\frac{1}{2}((\varphi+v) \tilde{\theta}, \psi)_{h}=(f, \psi)_{h}, \\
a(\theta, \psi)+\frac{1}{2}(\varphi \theta, \psi)_{h}=(f, \psi)_{h} \quad \forall \psi \in V_{h}(0),
\end{gathered}
$$

where $v \in H_{0}$. The relations (4.9) and (4.10) may also be written as

$$
\theta=S_{f}^{b}(\varphi), \quad \tilde{\theta}=S_{f}^{b}(\varphi+v) .
$$

Since $\varphi \in \Gamma$, there exists a unique $\theta$, with $\theta>0$ for $x>0$. We have the same properties for $\tilde{\theta}$, as soon as $\|v\|_{\infty ;(0,1)}$ is small enough, since for such a $v$, we have $\varphi+v \geq 0$.

Combining (4.9) and (4.10), we get

$$
a(\tilde{\theta}-\theta, \psi)+\frac{1}{2}(\varphi(\tilde{\theta}-\theta), \psi)_{h}=-\frac{1}{2}(v \tilde{\theta}, \psi)_{h},
$$

which implies

$$
\tilde{\theta}-\theta=S(\varphi) \cdot(-v \tilde{\theta})=-S(\varphi) \cdot v \theta-S(\varphi) \cdot v(\tilde{\theta}-\theta) .
$$


The solution $\tilde{\theta}-\theta$ of problem (4.11) satisfies

$$
\|\tilde{\theta}-\theta\|_{H^{1}(0,1)} \leq C\|v \tilde{\theta}\|_{h} \leq\|v\|_{\infty ;(0,1)}\|\tilde{\theta}\|_{h} .
$$

Moreover, using a discrete version of Poincaré's inequality,

$$
\|\tilde{\theta}\|_{h} \leq\|\tilde{\theta}\|_{H^{\prime}(0,1)} \leq C\|f\|_{h} \leq\|f\|_{C^{0}([0,1])},
$$

we get

$$
\|\tilde{\theta}-\theta\|_{H^{\prime}(0,1)} \leq C\|f\|_{C^{0}([0,1])}\|v\|_{\infty ;(0,1)} .
$$

Since $H^{1}(0,1)$ is included in $C^{0}([0,1])$, inequality (4.12) yields

$$
\|\tilde{\theta}-\theta\|_{\infty ;(0,1)} \leq C\|v\|_{\infty ;(0,1)}\|f\|_{C^{0}([0,1])} .
$$

Relation (4.13) implies that

$$
D S_{f}^{b}(\varphi) \cdot v=-S(\varphi) \cdot v \theta=-S(\varphi) \cdot v S_{f}^{b}(\varphi) .
$$

The definition of the operators $S_{f}^{b}(\cdot)$ and $S(\cdot)$ and the expression of $D S_{f}^{b}(\cdot)$ imply that $S_{f}^{b}(\cdot) \in C^{1}(\Gamma)$. Lemma 4.2 is thus proved.

Proof of Lemma 4.3. Let $\lambda$ be an eigenvalue of the operator $D S_{f}^{b}(w)$ and $p$ an associated eigenfunction,

$$
D S_{f}^{b}(w) \cdot p=\lambda p
$$

Using relations (4.6) and (4.15), we have

$$
-S(w) \cdot(p w)=\lambda p
$$

Let $q=-\lambda p$. From equation (4.7) we derive $q \in V_{h}(0)$ which satisfies

$$
a(q, \psi)+\frac{1}{2}(w q, \psi)_{h}=\frac{1}{2}(p w, \psi)_{h} \quad \forall \psi \in V_{h}(0) ;
$$

if $\lambda \neq 0$, we may also write

$$
a(q, \psi)+\frac{1}{2}(w(1+1 / \lambda) q, \psi)_{h}=0 \quad \forall \psi \in V_{h}(0) .
$$

Replacing $\psi$ by $\bar{q}$ (complex conjugate of $q$ ), we easily check that $(1+\lambda) / \lambda$ is real and $(1+\lambda) / \lambda<0$, that is, $-1<\lambda<0$.

To be more precise, let

$$
d_{1}=\inf _{z \neq 0 ; z \in V_{h}(0)} \frac{a(z, z)}{(w z, z)_{h}} .
$$

We may write

$$
-\frac{1+\lambda}{\lambda} \geq 2 d_{1} \text { or }|\lambda| \leq \frac{1}{1+2 d_{1}} .
$$

Since $d_{1} \geq \mu\|w\|_{\infty ;(0,1)}^{-1}$, we deduce inequality (4.8). Lemma 4.3 is thus proved.

\section{Miscellaneous Remarks}

Remark 5.1. The approximate problem has been studied in the case where $u(1, t)=b$, where $b$ is a given real number. The results obtained are still valid if $b$ is replaced by a function $t \mapsto b(t)$. 
Remark 5.2. For $b=0$, and more generally, for a dimension in space greater than one, with homogeneous Dirichlet boundary conditions, there exists an extinction time $T^{*}$, after which the solution is equal to zero. For more details, we refer to Le Roux [14] and to Berryman and Holland [5].

Remark 5.3. Hypothesis HP3 is essential for obtaining the error bounds.

To generalize the problem studied in the preceding sections, we may consider the case where $\gamma(s)=s^{p} / p, 3>p \geq 2$, and (or) the case where the spatial domain is, for example, a rectangle or a parallelepiped, located in the region $x_{1} \geq 0$. For such domains, we have to replace the boundary conditions by

$$
u(\vec{x}, t)= \begin{cases}0 & \text { for } x_{1}=0 \\ u_{1}(\vec{x}, t)>0 & \text { for } \vec{x} \in \partial \Omega \text { with } x_{1}>0\end{cases}
$$

where $\vec{x}=\left(x_{1}, \ldots\right)$, and we have to replace the hypothesis HP3 by $u_{0}(\vec{x}) \geq$ $\beta x_{1}$. We also assume some regularity and compatibility conditions for boundary and initial conditions corresponding to the hypotheses HP1 and HP2. In both cases, existence and uniqueness of a weak solution are still valid.

Remark 5.4. For a spatial dimension equal to two, a sufficient condition to get the existence and uniqueness of a positive approximate solution is that the discretization of the Laplacian satisfies the usual positiveness properties (for example, with triangular finite elements with three nodes, with the angles of the triangles being less than or equal to $\frac{\pi}{2}$ ). The proof of the error bounds is then extended in a straightforward way: we split the domain $\Omega$ in two parts $\Omega_{h}=\left\{\vec{x} \in \Omega ; 0<x_{1}<h\right\}$ and $\Omega_{h}^{c}=\left\{\vec{x} \in \Omega ; \vec{x} \notin \Omega_{h}\right\}$.

Remark 5.5. For the algorithm defined in $\S 4$, with $p=2$ and a spatial dimension at least 2 , the results stated in Lemmas $4.1,4.2$, and 4.3 are still valid. We have only to replace in the proof of Lemma 4.2 the inclusion of $H^{1}(0,1)$ in $C^{0}([0,1])$ by an inverse inequality,

$$
\|\tilde{\theta}-\theta\|_{\infty ; \Omega} \leq C(h)\|\tilde{\theta}-\theta\|_{H^{1}(\Omega)},
$$

and the results still hold, since, when using the algorithm, $h$ is a fixed parameter.

Remark 5.6. For the algorithm defined in $\S 4$ with $p>2$, we have to calculate the gradient of the operator $S_{f}^{b}(\cdot)$ defined by

$$
\varphi \rightarrow \theta=S_{f}^{b}(\varphi) \in V_{h}(b)
$$

where $\theta$ satisfies

$$
a(\theta, \psi)+\frac{1}{p}\left(\varphi^{p-1} \theta, \psi\right)_{h}=(f, \psi)_{h} \quad \forall \psi \in V_{h}(0) .
$$

We get

$$
D S_{f}^{b}(\varphi) \cdot v=-(p-1) S(\varphi) \cdot \varphi^{p-2} v S_{f}^{b}(\varphi),
$$

where $y \rightarrow \Psi=S(\varphi) \cdot y$ is defined by

$$
a(\Psi, z)+\frac{1}{p}\left(\varphi^{p-1} \Psi, z\right)_{h}=\frac{1}{p}(y, z)_{h} \quad \forall z \in V_{h}(0) .
$$


Then, for the spectral radius we get the estimate

$$
\rho\left(D S_{f}^{b}(w)\right) \leq(p-1) \frac{\left\|w^{p-1}\right\|_{\infty ;(0,1)}}{p \mu+\left\|w^{p-1}\right\|_{\infty ;(0,1)}},
$$

where $w$ is the fixed point of $S_{f}^{b}$ and $\mu$ is defined as in $\S 4$. The algorithm converges as soon as

$$
\mu\left\|w^{p-1}\right\|_{\infty ;(0,1)}^{-1}>1-\frac{2}{p}
$$

and, in view of the stability properties of the sequence $\left\{U^{n}\right\}$, this sufficient condition becomes

$$
\mu\left(\max \left(\left\|U^{0}\right\|_{\infty ;(0,1)}, b\right)\right)^{1-p}>1-\frac{2}{p} .
$$

For more details, we refer to [15].

\section{ACKNOWLEDGMENTS}

We are very grateful to the referee of this article for his very helpful advice and to Professor Ph. Benilan for the discussion we had about this problem.

\section{BIBLIOGRAPHY}

1. D. G. Arison and Ph. Benilan, Régularité des solutions de l'équation des milieux poreux dans $\mathbb{R}^{N}$, C. R. Acad. Sci. Paris Sér. A 288 (1979), 103-107.

2. D. G. Arison and L. A. Peletier, Porous medium equations, Nonlinear Analysis in Physical Sciences (J. Amman, ed.), Pitman, London, 1981.

3. Ph. Benilan, Opérateurs accrétifs et semi-groupes dans les espaces $L^{p}$, Functional Anal.Numerical Anal. Japan France seminar (H. Fuyita, ed.), Tokyo-Kyoto, 1976, pp. 15-52.

4.

5. J. G. Berryman and C. J. Holland, Stability of the separable solution for fast diffusion, Arch. Rational Mech. Anal. 74 (1980), 379-388.

6. Ph. Ciarlet, Introduction à l'analyse numérique matricielle et à l'optimisation, Masson, Paris, 1982.

7.

8. J. Dieudonné, Foundations of modern analysis, Academic Press, Paris, 1960.

9. J. R. Drake, J. R. Greenwood, G. A. Navratil, and R. S. Post, Diffusion coefficient scaling in the Wisconsin leviated octupole, Phys. Fluids 20 (1977), 148-154.

10. C. M. Elliott, Error analysis of the enthalpy method for the Stefan problem, IMA J. Numer. Anal. 7 (1987), 61-71.

11. A. Friedman, Partial differential equations of parabolic type, Prentice-Hall, London, 1960.

12. M. A. Herrero and M. Pierre, The Cauchy problem for $u_{t}=\Delta u^{m}$ when $0<m<1$, Trans. Amer. Math. Soc. 291 (1985), 147-158.

13. O. A. Ladyženskaja, V. A. Solonnikov, and N. N. Ural'ceva, Linear and quasi-linear equations of parabolic type, Transl. Math. Monographs, vol. 23, Amer. Math. Soc., Providence, RI, 1968.

14. M. N. Le Roux, Semi discretization in time of a fast diffusion equation, J. Math. Anal. Appl. 137 (1989), 354-370.

15. P. Lesaint and J. Pousin, Estimations d'erreur pour un problème parabolique dégénéré, Report, Dept. of Math., Swiss Federal Inst. of Technology, 1989.

16. R. H. Nochetto, Error estimates for two-phase Stefan problems in several space variables. I: linear boundary conditions, Calcolo 22 (1985), 457-499. 
17. R. H. Nochetto and C. Verdi, Approximation of degenerate parabolic problems using numerical integration, SIAM J. Numer. Anal. 25 (1988), 784-814.

18. O. A. Oleinik, The Prandtl system of equations in boundary layer theory, Dokl. Akad. Nauk SSSR 159 (1963), 583-586.

19. __ Sur certaines équations paraboliques dégénérescentes de la mécanique, Colloq. Internat. Equations aux Dérivées Partielles, CNRS, Paris, 1962.

20. E. S. Sabinina, A class of nonlinear degenerating parabolic equations, Soviet Math. 43 (1962), 495-498.

laboratoire de Calcul Scientifique, UFR Sciences, Université de Franche Comté, Route du Gray, F-25030 Besancon, France

Département de Mathématiques, Ecole Polytechnique Fédérale de Lausanne, CH 1015 LAUSANNE, SwitzerLAND 